\title{
Blade Segment with a 3D Lattice of Diamond Grits Fabricated via an Additive Manufacturing Process
}

\author{
Bin Chen ${ }^{1}$, Peng Chen ${ }^{1}$, Yongjun Huang ${ }^{1}$, Xiangxi Xu ${ }^{2}$, Yibo Liu ${ }^{3}$ and Shuangxi Wang ${ }^{1 *}$ (c)
}

\begin{abstract}
Diamond tools with orderly arrangements of diamond grits have drawn considerable attention in the machining field owing to their outstanding advantages of high sharpness and long service life. This diamond super tool, as well as the manufacturing equipment, has been unavailable to Chinese enterprises for a long time due to patents. In this paper, a diamond blade segment with a 3D lattice of diamond grits was additively manufactured using a new type of cold pressing equipment (AME100). The equipment, designed with a rotary working platform and 16 molding stations, can be used to additively manufacture segments with diamond grits arranged in an orderly fashion, layer by layer; under this additive manufacturing process, at least 216000 pcs of diamond green segments with five orderly arranged grit layers can be produced per month. The microstructure of the segment was observed via SEM and the diamond blade fabricated using these segments was compared to other commercial cutting tools. The experimental results showed that the 3D lattice of diamond grits was formed in the green segment. The filling rate of diamond grits in the lattice could be guaranteed to be above $95 \%$; this is much higher than the $90 \%$ filling rate of the automatic array system (ARIX). When used to cut stone, the cutting amount of the blade with segments made by AME100 is two times that of ordinary tools, with the same diamond concentration. When used to dry cut reinforced concrete, its cutting speed is $10 \%$ faster than that of ARIX. Under wet cutting conditions, its service life is twice that of ARIX. By applying the machine vision online inspection system and a special needle jig with a negative pressure system, this study developed a piece of additive manufacturing equipment for efficiently fabricating blade segments with a 3D lattice of diamond grits.
\end{abstract}

Keywords: Diamond blade segment, Additive manufacturing, 3D lattice of diamond grits, Cold pressing equipment, Machine vision inspection

\section{Introduction}

Diamond grit has been widely used in machining tools owing to its outstanding hardness, sharpness, and thermal conductivity performance [1]. Generally, the diamond grits are mechanically mixed with metal powders for fabricating diamond tools. Zhang et al. [2] and Zhou et al. [3] proposed that under the condition of high-speed

\footnotetext{
*Correspondence: sxwang@stu.edu.cn

${ }^{1}$ Intelligent Manufacturing Key Laboratory of Education, Department of China, College of Engineering, Shantou University, Shantou 515063, China

Full list of author information is available at the end of the article
}

cutting in modern precision machining, traditional diamond tools become obsolete owing to low cutting efficiency and heavy consumption. Roshon [4] fabricated a type of electroplated diamond tool that has a much higher machining efficiency at the same cutting speed, compared to traditional diamond tools. Zhang [5] electroplated diamond grits in an orderly arrangement on the surface layer of a drill bit via the dispensing method; eventually obtaining a $30 \%$ increase in drilling efficiency and an $85 \%$ extended service life. However, diamond grits on electroplated diamond tools are bonded mechanically at their thin coating base and are easily pulled off during 
machining [6]. To improve the service life, Chattopadhyay [7] fabricated monolayer brazed diamond tools to achieve chemical bonding between the diamond grits and matrix, in which diamond grits were embedded firmly in the brazed additive matrix. Yu et al. [8] compared the monolayer brazed and sintered diamond tools used for grinding stone and found that both bonding force and protrusion height of the brazed diamond tool improved smoothly throughout the grinding process. Bonding force can be strengthened via the addition of some active elements, such as $\mathrm{Ni}$ and $\mathrm{Ti}$, in the brazing alloy by forming a discontinuous transitional carbide layer during the brazing process $[9,10]$. Furthermore, the diamond interval determines the cutting time for each grit and the space for cutting debris to exit. The grit protrusion affects the cutting speed of the diamond tool. According to the results of sawing experiments, Dong et al. [11] proposed that a reasonable protrusion height, proper interval, and reasonable matching of diamond grits and bonding matrix played key roles in obtaining surface uniformity of the saw tooth and service life of the saw. Sung brazed diamond grits onto surface layers of wire saw in an orderly fashion by placing multiple diamond grits in a ceramic mold with a predetermined pattern. Compared to conventional diamond wire saws, brazed diamond grits in Sung's process were arranged in an orderly fashion on the surface layer and protruded twice as high as that of mechanically mixed diamond grits; these could withstand aggressive cutting without pulling off, attaining approximately one to three times faster cutting speeds $[12,13]$. Burkhard et al. [14] sprayed the grits on glue droplets that had been arranged in a controlled fashion on the surface of a rotary steel substrate to fabricate the honing tool. The monolayer brazed diamond honing tool containing orderly arranged diamond grits services 10 times longer than that of the electroplated diamond tool with the same honing process parameters.

Unfortunately, neither the brazed nor electroplated diamond tool demonstrated both high sharpness and long service life under the conditions of ultra-precision machining. Once the monolayer diamond grits had worn out, the whole diamond tool would fail to successively supply diamond grits and required scrapping. Wu et al. [15] produced a multilayered brazed diamond wheel via a combination of compressed molding and vacuum brazing, significantly improving the bonding strength and service life. Song et al. [16] developed multilayer brazed diamond saw blades through a liquid phase sintering process and found that the sharpness only increased by $22.3 \%$. The disadvantages of the poor self-sharpening ability of multilayer brazed diamond tools are due to their casting microstructure with a poor grindability, which makes it difficult for the diamond grits to protrude from the bonding matrix [17]. Moreover, the diamond grits could be graphitized during the multilayer brazing process, although this can be prevented by treatment with vacuum and argon gas [18]. In 2005, Shinhan Diamond developed a kind of automatic equipment known as the automatic array system (ARIX) to precisely place diamond grits in 3D patterns, as seen in Figure 1. It was reported that ARIX, which encased the diamond grit with glue and metal powders, forming a ball, could produce up to 50000 pcs of green segments with a 3D lattice of diamond grits per month by cold pressing the balls into a segment [19]. The diamond tool with a 3D lattice of diamond grits is solid sintered at a relatively low temperature and has the advantages of both sharpness and long service life. To improve productivity, in 2011 Dr. Fritsch, a pioneering diamond tools manufacturing company, had developed its own equipment, the KPV300, with two manipulators; this could produce approximately 100000 pcs of green segments with a 3D lattice of diamond grits per mouth. However, the placing accuracy of diamond grits (90\%) by KPV300 is much lower than that by ARIX (98\%) because of the wider range of diamond grit placement by the manipulators.

Recently, additive manufacturing technologies have been widely applied in the industry owing to the possibility of efficiently fabricating realistic looking and complex parts by advanced digital control technology [20]. Plocher [21] summarized the superior advantages of additive manufacturing as structure optimization, freeform fabrication, and rapid prototyping. Aided with some commercial software, such as topology optimization, computationally inexpensive solutions can be easily realized in industrial manufacturing. In 2016, Yang et al. [22] fabricated a monolayer diamond grinding wheel with a regular grit distribution based on 3D printing technology, providing a solution to the complicated preparation process. It was reported that the diamond grits did not fall off, even after the grinding processes involving a heavy

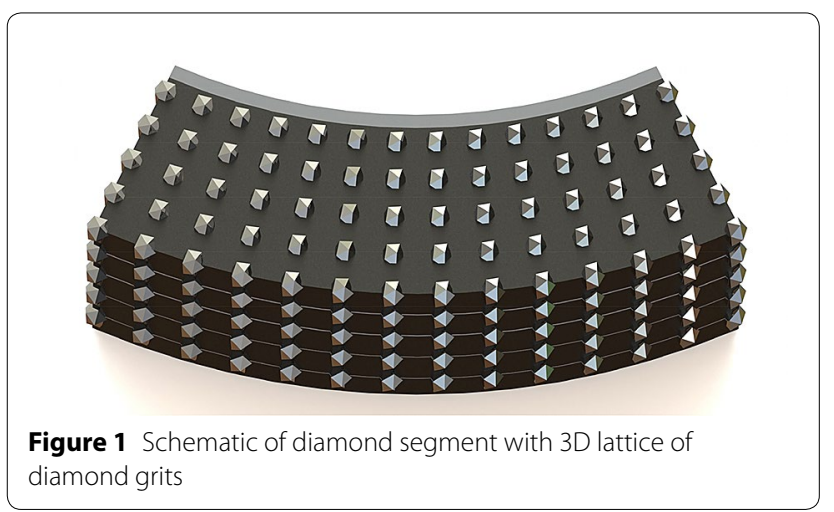


load. In addition, in 2017 Yang tentatively manufactured a multilayer diamond tool via 3D printing technology [23]. First, the diamond grits were randomly sprayed onto alloy powders and sintered by a laser beam, according to the design requirements; the diamond grits that were not sintered were wiped away using a sparger. Material accumulation then occurred via layer-by-layer sintering. As a result, none of the diamond grits fell off under normal use. However, compared to the ARIX or KPV300, this $3 \mathrm{D}$ printing process currently has much lower productivity. To date, diamond blades with ordered diamond grits manufactured by ARIX are the mainstream choice in the worldwide market.

To significantly improve production efficiency and performance of the diamond tool, a diamond blade segment with a 3D lattice of diamond grits was manufactured using a new type of additive manufacturing equipment, with a rotary working platform (AME100). The rotary working platform is divided into 16 stations and every three successive stations fabricate a layer of orderly arranged diamond grits. At least 216000 pcs of green segments with a 3D lattice of diamond grits could be produced per month, a production efficiency more than two times that of the KPV300. The AME100 features a special needle jig with inner holes and a negative pressure system, which arranges diamond grits by rule and line in metal bonding agent powders. Meanwhile, benefitting from the machine vision online inspection system, an adsorption rate of diamond grits as high as $98 \%$ can be guaranteed, with the diamond grits arranged at equal distances from one another in the green segment.

\section{Preparation of Green Segment with 3D Lattice of Diamond Grits}

\subsection{Principle of Arranging Diamond Grits in Order}

The orderly arrangement of diamond grits has been an area of significant interest in the machining field. For example, in 1999, Gåhlin successfully arranged diamond grits on an abnormal surface in a regular pattern via duplicating diamond grits onto a silicon wafer, based on hot filament chemical vapor deposition; the shape, size, and packing pattern of the pyramids had been defined by photolithography and etching [24].

In the additive manufacturing process, to ensure arrangement of the diamond grits in a certain direction and at equal distances from one another, a needle jig with inner holes and a negative pressure system was designed to absorb the diamond grits. The inner holes were designed to be smaller than the average diameter of the diamond grits so that the diamond grit could be absorbed but not drawn into the needle hole. As shown in Figure $2 \mathrm{a}$, the adsorption process is as follows: first, the needles arranged in the dip mold are located at the level of the diamond grit and each needle absorbs one diamond grit via negative pressure from a vacuum. The diamond grits absorbed by the needle jig are subsequently brought above the metal powders and located exactly at the prepressed metal bonding powders; the vacuum state of the needle jig is then turned off. Even if a diamond grit gets stuck in the needle hole, it will be squeezed out when the needle recedes from the dip mold. Therefore, the ordered distribution of diamond grits can be achieved in one layer. By feeding the metal powders and diamond grits in turn, a 3D lattice of diamond grits will be formed in the green segment.

\subsection{Guaranteeing Filling Rate of Diamond Grits in Segment} Human vision and thinking can be simulated by machine vision by using optical systems, industrial cameras, and image processing technologies [25]. With the advantages of high positioning accuracy and processing speed, machine vision provides innovative solutions in numerous industrial manufacturing processes [26]. Machine vision can be used to easily measure 3D sizes and detect defects in machine parts $[27,28]$. In the welding process, Sun et al. [29] proposed an effective machine vision online inspection method that showed an accuracy of more than $99 \%$. However, spatter formation is still an issue in welding. Schweier et al. [30] developed a machine vision system for tracking spatter using highspeed cameras. In recent years, machine vision has also been applied for object detection and size measurement in grinding or harvesting robots [31, 32]. In addition, with the application of deep learning methods, machine vision becomes more and more intelligent for industrial production [33].

During an absorbing diamond grits process, factors such as abrasion of needle inner hole, fluctuation of vacuum negative pressure, and irregular shaped diamond grits, may result in accidental failure of the absorbing diamond grit process. This uncertainty in the grit absorbing rate affects the uniformity and filling rate of diamond grits in every layer of the green segment. Thus, a machine vision online inspection system was introduced in the fabrication process to ensure a consistent adsorption rate by learning the standard product, extracting location parameters from images, and processing it to obtain a desired form.

As opposed to taking photographs from above in most cases of machine vision, the camera in this research is fixed under a loading device (AME100) and captures images in a bottom-up mode, as shown in Figure 3. In this manner, the skip car moves below the needle jig bottom and ascends up. Thereafter, the needles with inner holes absorb the diamond grits. When the skip car withdraws from the bottom of the needle jig to the original 


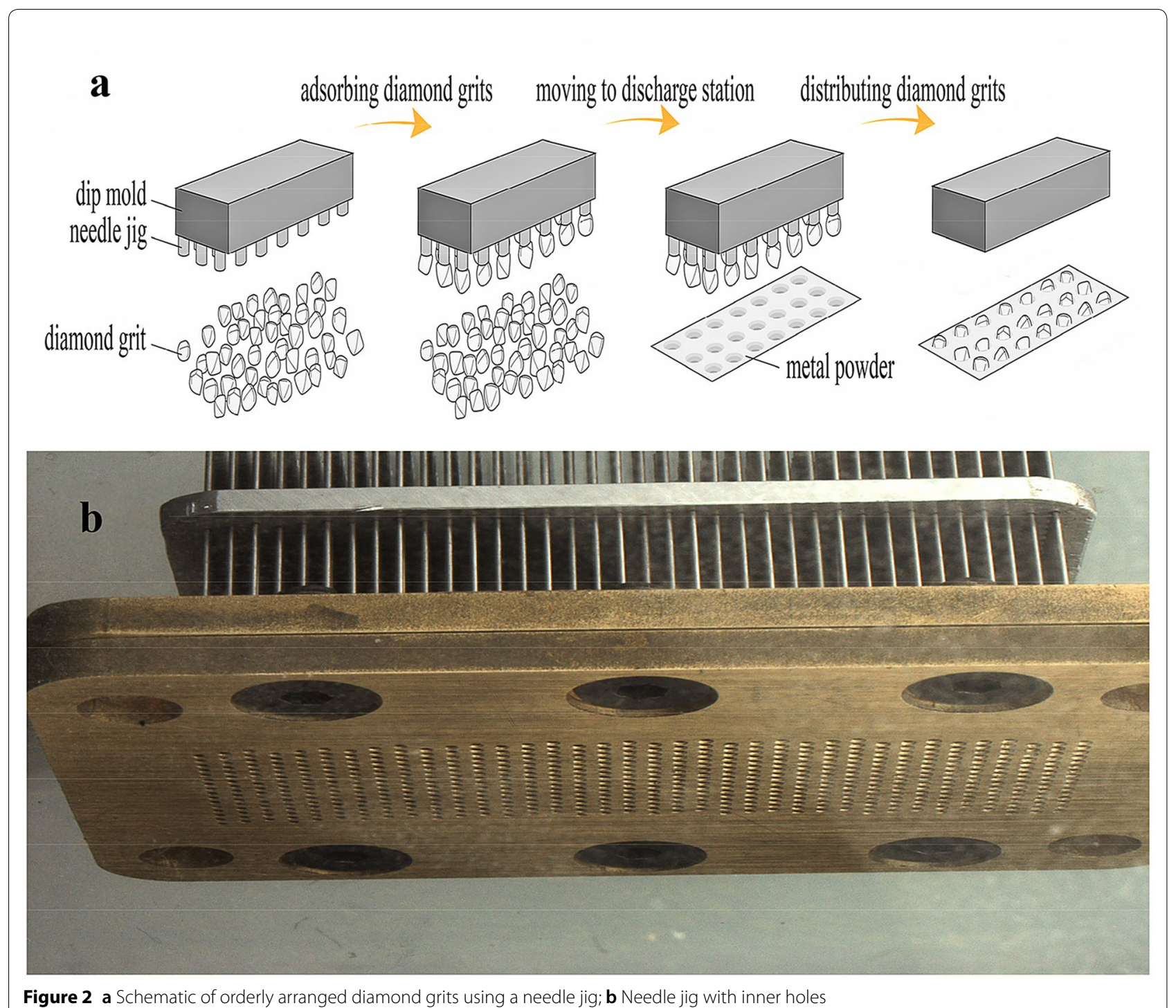

position, an image of absorbing status is captured by the camera (BASLER, Germany) in 0.1s. Finally, the adsorption rate is calculated. Due to the special structure design of the machine vision online inspection system, it does not prolong the running time for fabricating the diamond green segments using the 3D lattice of diamond grits.

The lighting system weakens the chaos and amplifies the image features and defects [34]. Diamond grit as a single crystal always results in special reflective phenomena due to its anisotropy, because of which it is often misjudged as a vacancy hole when an ordinary flat panel light source is used. According to the mechanical structure of AME100, two white strip LED light sources are set symmetrically at $60^{\circ}$ to illuminate the needle jig, because the scattered light produced by the LEDs can optimize image quality at specific angles. Under this lighting system, the boundary between the hole position area and the background is clear, and thus, the misjudgment can be eradicated, as seen in Figure 4.

The machine vision system in this research combined several kinds of algorithms, such as the product learning algorithm, the improved position features extraction algorithm, and the data mining algorithm, to classify the decision tree. By calculating the variance between the gray value of one hole and the average value of the whole area, the machine vision inspection system could judge in turns if one hole was filled or empty with diamond grit. In addition, to avoid the influence of abrasion loss of needle hole, real-time image subtraction was applied in the feature extraction process. Combining the 

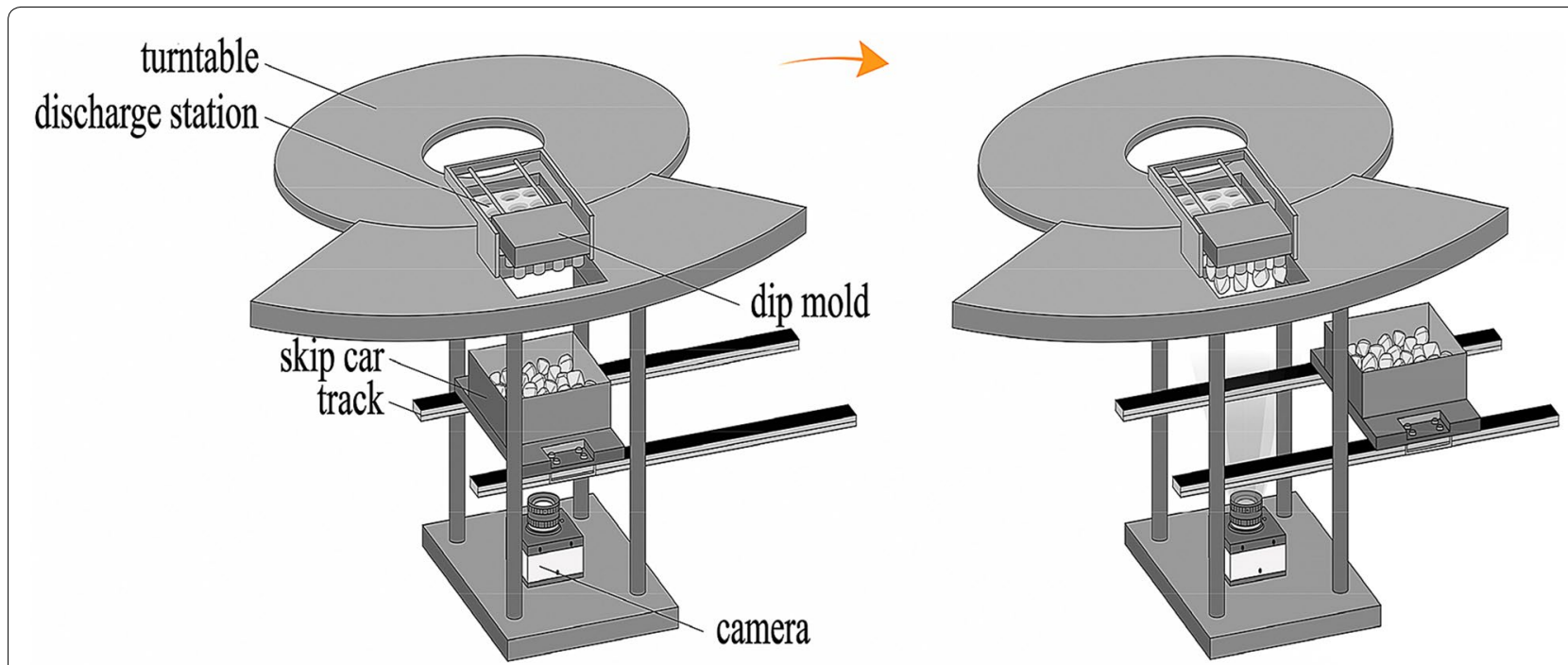

Figure 3 Schematic of online detection of diamond grit adsorption rate

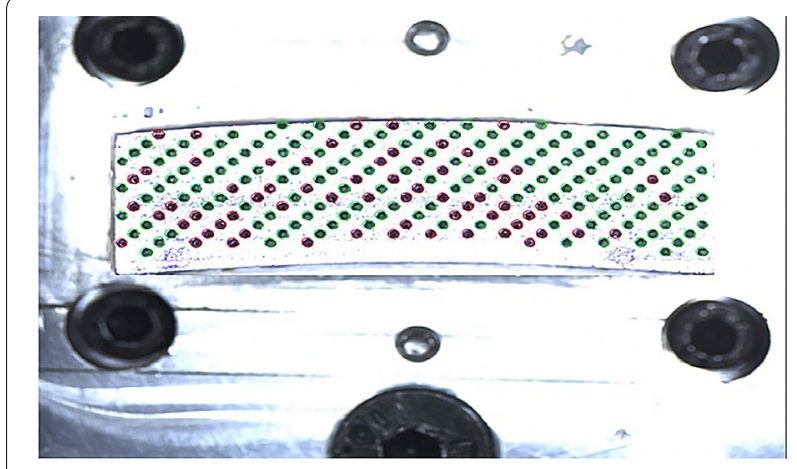

Figure 4 Image processing result of the machine vision system

aforementioned algorithms, the absorption status of diamond grits by the needle jig could be judged accurately. In Figure 4, the green shows when the diamond grit was absorbed successfully in a needle hole, and the red shows when it was not.

\subsection{Additive Manufacturing Process of Segment with 3D Lattice of Diamond Grits}

As a rapid prototyping technology, additive manufacturing has great potential in the manufacturing of diamond tools. Based on the principle of additive manufacturing, a type of rotary working platform was designed. As shown in Figure 5, the rotary disk is divided into 16 stations, including the loading station, ordered arrangement station, cold pressing station, and ejection station, and every three successive stations form a layer with orderly arranged diamond grits. In the first station, the metal powders are fed

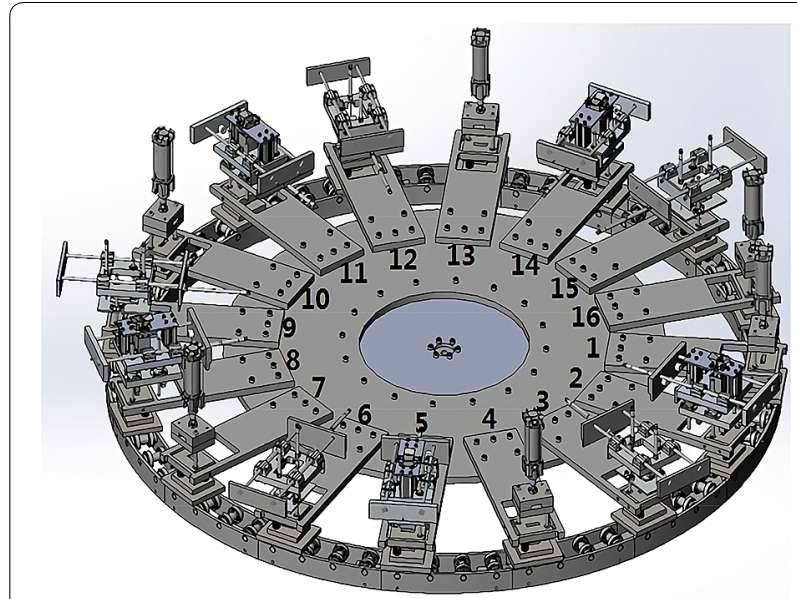

Figure 5 Schematic of rotary working platforms: 1, 4, 7, 10, 13. Stations for loading metal powders; $2,5,8,11,14$. Stations for ordered arrangement of diamond grits; $3,6,9,12,15$. Stations for cold pressing; 16. Station for segment ejection

into the mold cavity and pressed using a special mold to form ordered pits. The rotary disk then rotates from station 1 to station 2, where the needle jig with diamond grits is located, as shown in Figure 6. When the mold cavity reaches station 2 , the diamond grits absorbed by the jig, which were detected by the machine vision online inspection system fixed under station 2, are placed on the corresponding pits in the metal powders. Next, the rotary disk rotates to station 3 , and the diamond grits and metal powders in mold cavity are compacted to form a layer with orderly arranged diamond 

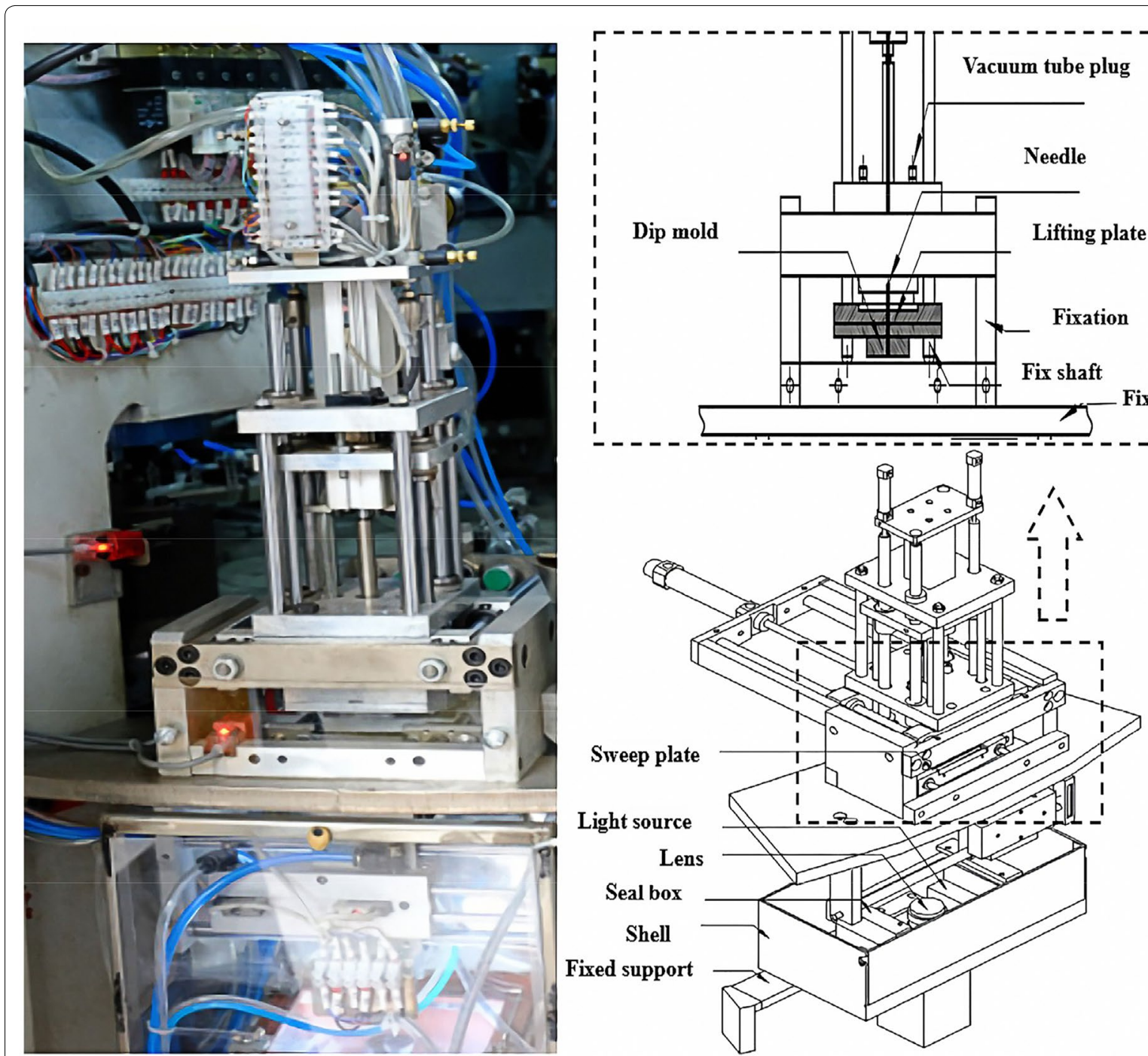

Figure 6 Schematic of station 2 with online inspection system and device for ordered arrangement of diamond grits

grits. Thereafter, the rotary disk successively rotates from station 4 to station 15 and repeats the above three steps of stations 1 to 3 in a circular manner. Using this process, the green diamond segment with 3D lattice of diamond grits was additively manufactured layer by layer. When the rotary disk reaches station 16 , the green segment consisting of five layers with orderly arranged diamond grits are collected by a transport belt.

The theoretically designed upper speed of the rotary disk is $6.25 \mathrm{r} / \mathrm{min}$. In actual production, the equipment operates at $5 \mathrm{r} / \mathrm{min}$, which means that at least 216000 pcs of segments with orderly arranged diamond grits could be produced per month via the additive manufacturing process, which exceeds the production using the KPV300 or ARIX system.

\section{Experiments and Discussion}

Using the new equipment AME100, diamond blade segments with a 3D lattice of diamond grits were additively manufactured. As shown in Figure 7a, the AME100 is under production at Advanced Technology \& Materials Co., Ltd., China. As seen in Figure 7b, the diamond grits are arranged in an orderly manner on the surface of the blade after applying the diamond segments produced by the AME100. The characteristics of the AME100 and its diamond segment products were compared with those of the ARIX and KPV300. 

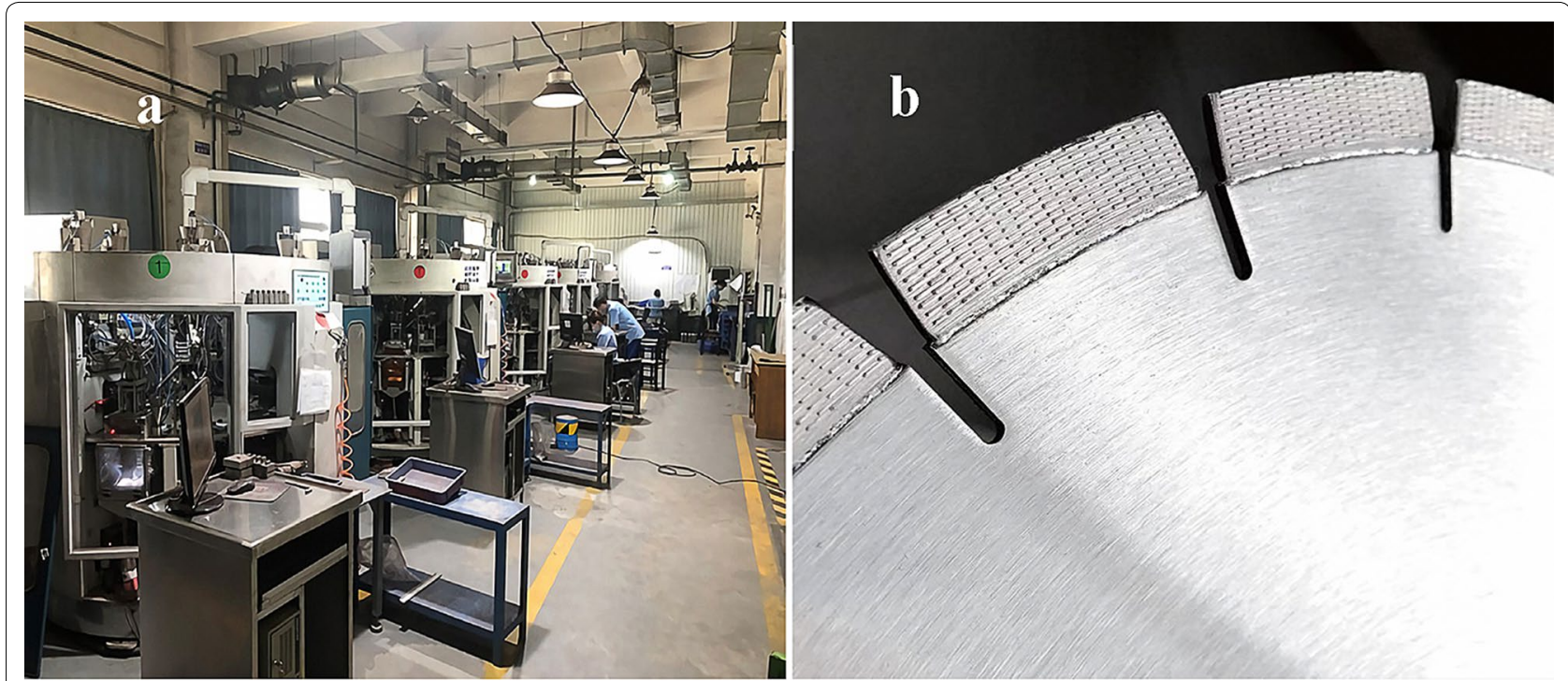

Figure 7 AME100 equipment and its segment product: a workshop with AME100; b surface morphology of diamond segment using AME100

Table 1 Detection accuracy of the inspection system at station 5 for different needle numbers

\begin{tabular}{llll}
\hline Needle number & $\begin{array}{l}\text { Counted } \\
\text { by camera }\end{array}$ & Counted visually & $\begin{array}{l}\text { Detection } \\
\text { accuracy } \\
\text { (\%) }\end{array}$ \\
\hline 260 & 241 & 241 & 100 \\
260 & 247 & 247 & 100 \\
260 & 244 & 244 & 100 \\
370 & 351 & 351 & 100 \\
370 & 352 & 352 & 100 \\
370 & 354 & 354 & 100 \\
\hline
\end{tabular}

\subsection{Detection Accuracy of Machine Vision Online Inspection System}

To verify the compatibility of the machine vision online inspection system for different absorbing needle numbers, the accuracy was tested for two sets of three batches of data. To confirm the accuracy under the complex distribution conditions of diamond grits, the diamonds absorbed on the jig were deliberately maintained as inadequate. On one hand, the amount of diamond grits absorbed by the jig was provided by the machine vision online inspection system. On the other hand, the real number was manually counted by visual inspection according to the images captured. The experimental results demonstrate that regardless of the number of needles in the jig (i.e., 260 or 370), the system accuracy was maintained as $100 \%$ at station 5 , as shown in Table 1. Therefore, it can be concluded that the machine vision online inspection system exhibits effective compatibility with the working process.

\subsection{Microstructure of the Segment with 3D Lattice of Diamond Grits}

The microstructures of the green and sintered segments were observed by scanning electron microscopy (SEM). Figure $8 \mathrm{a}, \mathrm{b}$ show the secondary electron SEM images of the green segment with 3D lattice of diamond grits for the cutting blade produced by the AME100. It can be seen that the diamond grits are embedded half in the bonding metal agent and are arranged regularly. Figure $8 \mathrm{~b}$ is the cross-sectional morphology of the green segment. Because this product has not been sintered, the bonding strength of the green segment is weak. Therefore, when it was broken off through knocking with a hammer, the fractured surface appeared uneven. The diamond grits are observed to disperse in a disorderly manner because they are not at the same latitude in the green segment. However, the outlines of the five diamond grit layers are still retained to a certain extent if the two surface layers of the green segment are also considered. Moreover, the diamond grit layers are maintained parallel to each other. This means that the diamond grits could not only be arranged in order in a single layer but also form a diamond grit lattice in 3D space through the additive manufacture process using the proposed equipment.

Figure $8 \mathrm{c}-\mathrm{f}$ show the EDS analysis results of the fracture morphology. The EDS image of $\mathrm{C}$ represents the distribution of the diamond grits in the bonding agent. As can be seen from Figure 8c, d, although some of the diamond grits are pulled out during the fracture process, it is obvious that the diamond grits are arranged regularly in the green segment. The EDS images of $\mathrm{Cu}$ and Fe represent the distributions of metals in the segment. As 

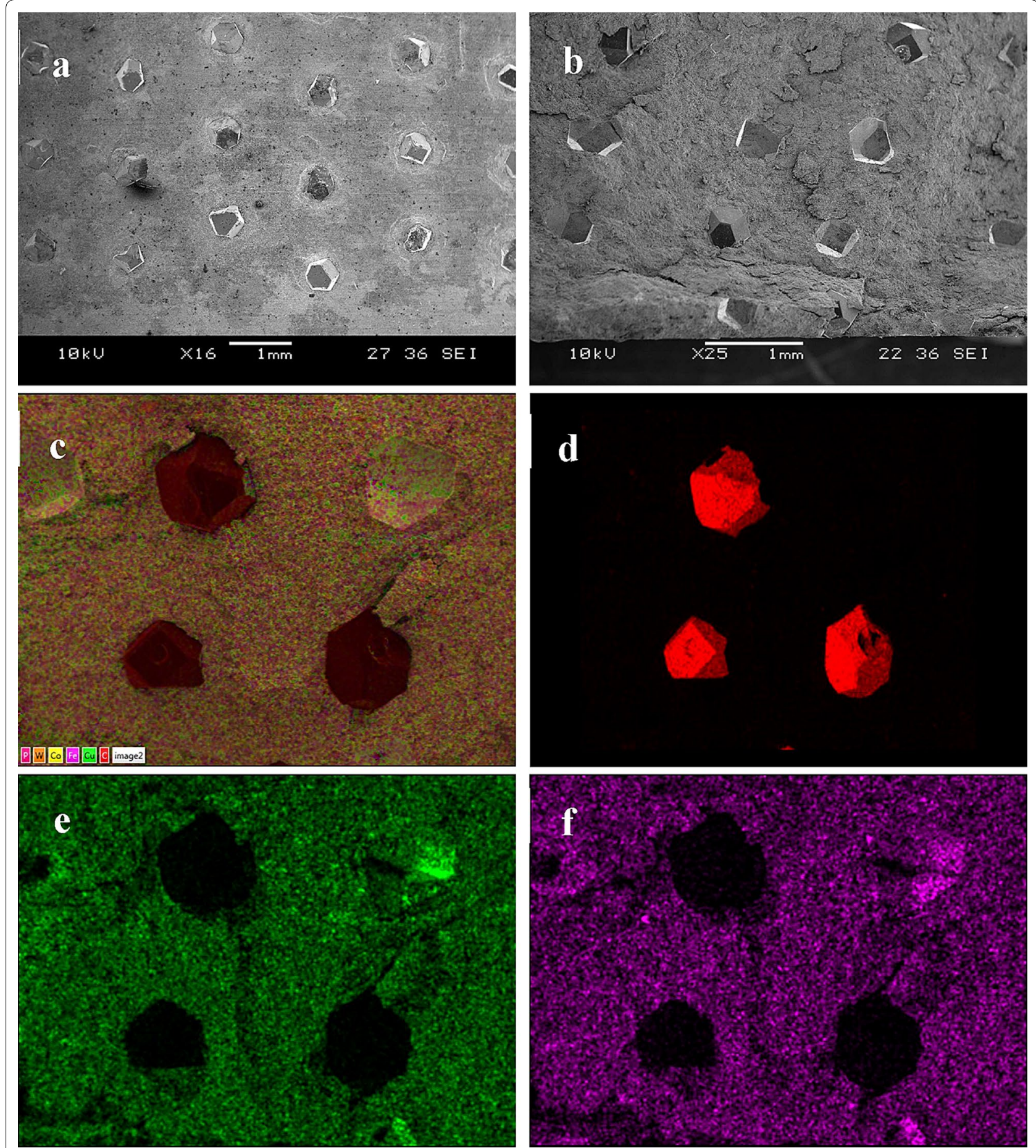

Figure 8 Microstructure of the segment with 3D lattice of diamond grits: a surface of the green segment; $\mathbf{b}$ cross-sectional surface of the green segment; $\mathbf{c}$ EDS hierarchical image of $\mathbf{b}$; $\mathbf{d}$ EDS of $\mathrm{C}$ from $\mathbf{c}$; $\mathbf{e}$ EDS of Cu from $\mathbf{c} ; \mathbf{f}$ EDS of Fe from $\mathbf{c}$

shown in Figure 8e, $\mathrm{f}$, the elements are distributed somewhat homogeneously in the bonding base by the prealloyed-powder additive manufacture process. This could guarantee optimal cutting performance of the diamond segment in terms of the bonding agent base.

The morphology of a segment from a used diamond drill tool was also investigated. As can be seen from the 
fracture surface microstructure in Figure 9, the SMD diamond grits have excellent crystal structure, and the grits seem to be arranged in a disorderly manner on the fracture surface of the ductile metal bonded base owing to the fact that they are not at the same latitude. After drilling stone with the tool for one day, the ordered arrangement of diamond grits on the drill tool's surface layer present a different morphology. As shown in Figure 9b, the diamond grits are arranged in an orderly manner and solidly embedded in the sintered metal base of the segment. Some diamond grits have been broken, and some have been ground during the drilling process because of their different crystal orientations in the drill tool matrix. When compared with that of brazed diamond tools, the good grindability of the solid sintering metal base of these sintered diamond tools is conducive to timely diamond shedding, revealing new cutting edges [17]. The successive breaking of diamond grits on the drill surface helps to maintain the balance between sharpness and service life. Furthermore, the strength of the solidstate sintered matrix in the blade segment produced via AME100 is much higher than that of liquid-phase sintering process involving the brazing diamond tools. The consolidation strength of diamond grits and wearability of the matrix can then be improved at the equilibrium state, which enables the drill tool to withstand high speed machining well. The surface morphology of the used drill proved that the diamond tool containing segments with 3D lattices of diamond grits would exhibit favorable selfsharpening functions and a long service life during the machining process.

\subsection{Performance of Equipment and Diamond Blades with Orderly Arrangement of Diamond Grits}

To date, ARIX, KPV300, as well as AME100, have been able to automatically enable fabrication of green segments with 3D lattices of diamond grits. The characteristics of these equipment and their segment products were
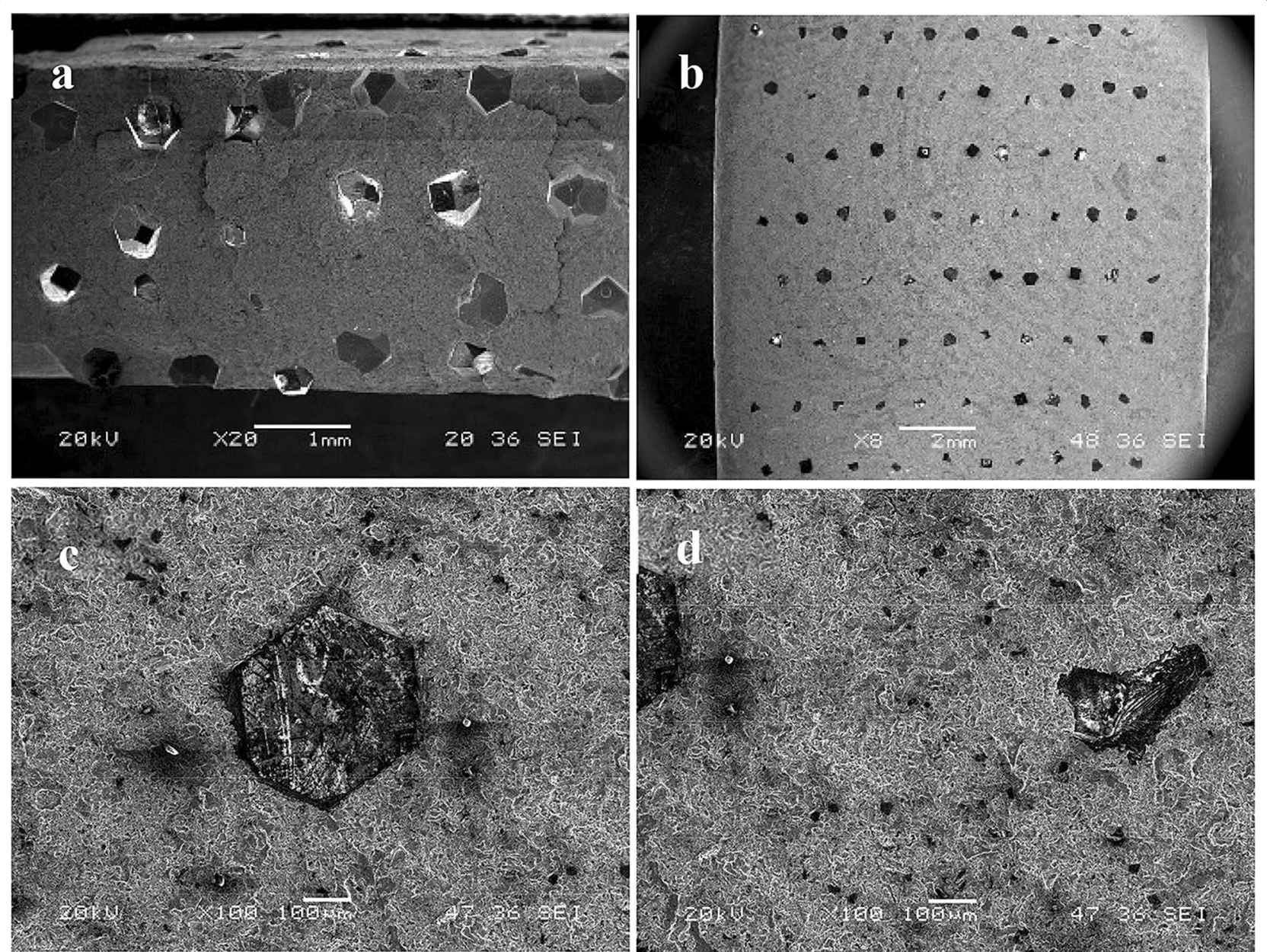

Figure 9 Morphology of used diamond drill with 3D lattice of diamond grits: $\mathbf{a}$ fracture surface; $\mathbf{b}$ surface layer after grinding; $\mathbf{c}$ regular grit in $\mathbf{b}$ after grinding; $\mathbf{d}$ broken grit in $\mathbf{b}$ after grinding 
Table 2 Performance comparison of different equipment for diamond green segments

\begin{tabular}{|c|c|c|c|}
\hline & AME100 & ARIX & KPV300 \\
\hline Equipment structure & Rotating multi-stations & Single station & Single station \\
\hline Machine vision inspection & Yes & No & No \\
\hline Production efficiency (pcs/month) & 216000 (5 layers) & 50000 (5 layers) & 120000 (5 layers) \\
\hline Filling rate $(\%)$ & $>95$ & $80-90$ & 90 \\
\hline Inspection accuracy (\%) & 98 & 98 & 90 \\
\hline Product yield $(\%)$ & $>95$ & 80 & 80 \\
\hline Maximum concentration (\%) & 60 & 30 & 50 \\
\hline
\end{tabular}

Table 3 Comparison of blade with 3D lattice of diamond grits and ordinary commercial blade

\begin{tabular}{lll}
\hline & Blade with 3D lattice & Ordinary blade \\
\hline $\begin{array}{l}\text { Diamond grits concentration } \\
(\%)\end{array}$ & 20 & 20 \\
$\begin{array}{l}\text { Machine model } \\
\text { Motor power (kW) }\end{array}$ & HSM-600 & HSM-600 \\
$\begin{array}{l}\text { Cutting material hardness (HS) } \\
\begin{array}{l}\text { Maximum cutting speed (m/ } \\
\text { min) }\end{array}\end{array}$ & 89.5 & 15 \\
Load current (A) & 35 & 89.5 \\
Cutting amount (m) & 3200 & 2.8 \\
\hline
\end{tabular}

analyzed at Advanced Technology \& Materials Co., Ltd. Beijing, which is one of the leading diamond tool manufacturing enterprises in China. The performances of the abovementioned equipment-after having undergone thousands of tests-are shown in Table 2. Owing to the multi-station rotating structure and the machine vision on-line inspection system, the production efficiency of AME100 is as high as that achieved with 216000 pcs of green segments with five layers per month, which is about five times that of ARIX and twice that of KPV300. More importantly, the green segments fabricated via
AME100 are better in quality than those fabricated via the other equipment; for example, the former show much higher filling rates and location accuracies of the diamond grits. In particular, the product yield of AME100 exceeds $95 \%$, which is significantly superior to that of ARIX or KPV300.

Through the experiment involving the cutting of the stone ( $2 \mathrm{~cm}$ thick, produced in Cenxi, China), the diamond blade containing the segment produced via AME100 was compared with a conventional blade of equal thickness with randomly distributed diamond grits at the same diamond concentration. As indicated in Table 3, the cutting speed of the diamond blade with the 3D lattice could attain a value up to $6 \mathrm{~m} / \mathrm{min}$, which is twice that of the conventional diamond blade. The amount of stone cut doubled at the same diamond grit concentration.

Furthermore, the diamond blades with segments manufactured via AME100 and ARIX were used to cut concrete reinforced by four steel bars of $\Phi 8 \mathrm{~mm}$, under wet or dry conditions respectively; the performances thereof were compared. From Table 4, it can be seen that when the road concrete materials were cut under dry conditions, the cutting speed of the LW350-AME100 was nearly $10 \%$ higher than that of the LW350-ARIX, and the wear height loss of these two blades was the same. When

Table 4 Experiment involving cutting of reinforced concrete under different condition

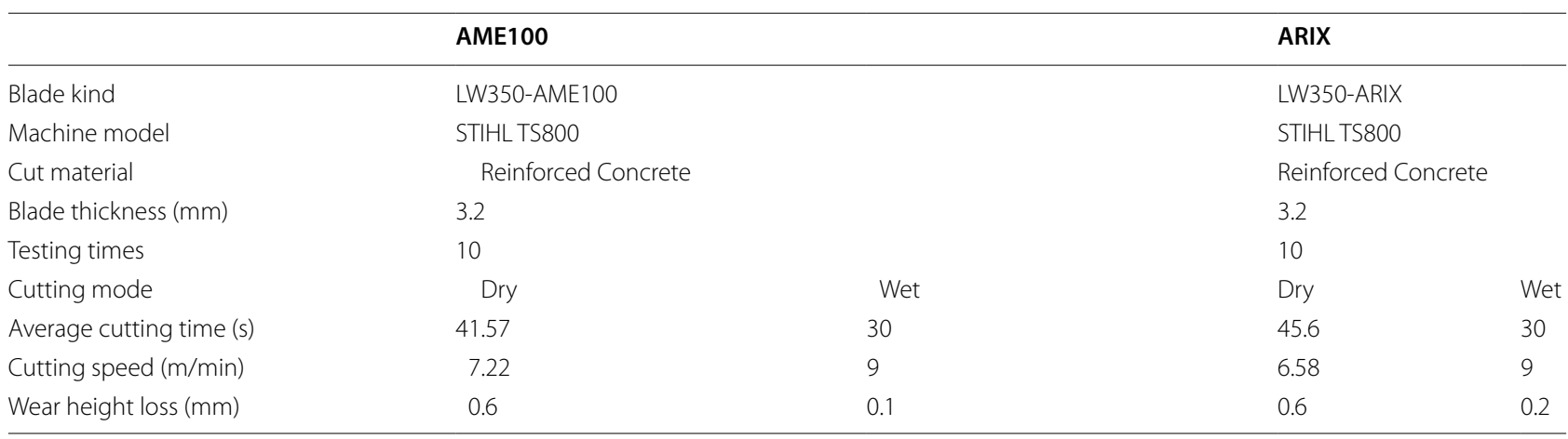


the road concrete materials were cut under wet conditions, the height loss of the LW350-AME100 was half that of the LW350-ARIX for the same cutting speed.

\section{Conclusions}

In this study, a diamond blade segment with a 3D lattice of diamond grits was additively manufactured via a new kind cold press equipment, namely AME100.

(1) Through the application of a machine vision online inspection system and a special needle jig with a negative pressure system, the diamond grits were absorbed and arranged in an orderly manner in the metal bonding agent powders to form one layer of the diamond green segment. Moreover, a diamond grit filling rate above $95 \%$ in the green segment could be guaranteed.

(2) With a rotary working platform and 16 molding stations of AME100, at least 216000 pcs of diamond green segments with five layers and orderly arranged grits could be produced per month, which shows a much higher efficiency compared with that of advanced equipment such as ARIX or KPV300.

(3) A microstructure observation showed that the diamond grits of the segments were arranged at equal intervals in three dimensions. The 3D lattice of the diamond grits was formed in the diamond segment.

(4) The cutting amount achieved with the diamond blade with segments fabricated via AME100 was twice that achieved with ordinary diamond tools for the same diamond concentration, when they were used to cut stone. For cutting reinforced concrete under dry conditions, it achieved a cutting speed $10 \%$ faster than that of the ARIX blade. For cutting reinforced concrete under wet conditions, its service life was twice that of the ARIX blade.

\section{Acknowledgements \\ The authors sincerely thank Prof. Yuejuan Lin for her help in microstructure observation and senior engineer Xiaoli Luo for providing cutting data of diamond tools.}

\section{Authors' Contributions}

BC assisted the design and manufacturing of AME100, collected experiments' date and wrote papers; PC analyzed the microstructure of blade segment with 3D lattice of diamond grits; $\mathrm{YH}$ took part in the design of machine vision inspection system; XX proposed and manufactured the equipment with rotary working platform; YL produced segments with AME100 and tested performance of the cutting tools; SW conceived the machine vision online inspection system to realize the stable filling rate of diamond grits by AME100. All authors read and approved the final manuscript.

\section{Authors' Information}

Bin Chen, born in 1996, master candidate at Intelligent Manufacturing Key Laboratory of Education Department of China, Shantou University, China. His research interests involving mechanical manufacture and automation.
Peng Chen, born in 1993, got his master degree from Intelligent Manufacturing Key Laboratory of Education Department of China, Shantou University, China, in 2020. His research interests involving mechanical manufacture.

Yongjun Huang, born in 1992, received his master degree from Shantou University, China, in 2018. His research interests involving mechanical engineering.

Xiangxi Xu is currently a general manager at Shantou Yuexi Diamond Tools Co., $L t d$. His research interests involving cold pressed equipment.

Yibo Liu, born in 1966, senior engineer. He received his doctoral degree from China University of Geosciences, China, 1997. His research interests involving powder metallurgy and superhard tool.

Shuangxi Wang, born in 1965, professor at College of Engineering, Shantou University, China. He received his doctoral degree from Tsinghua University, China, in 2002. His research interests involving precise manufacturing \& light industrial equipment.

\section{Funding}

Supported by Scientific Research Funds of Guangdong Province of China (Grant No. 2017B090922008), and the Scientific Project of Chaozhou of China (Grant No. 2018ZD10).

\section{Competing Interests}

The authors declare no competing financial interests.

\section{Author Details}

${ }^{1}$ Intelligent Manufacturing Key Laboratory of Education, Department of China, College of Engineering, Shantou University, Shantou 515063, China

2 Shantou Yuexi Machinery Equipment Co. Ltd, Shantou 515000, China.

${ }^{3}$ Advanced Technology \& Materials Co., Ltd, Beijing 100081, China.

Received: 27 January 2020 Revised: 5 July 2020 Accepted: 14 October 2020

Published online: 28 October 2020

\section{References}

[1] VI Lavrinenko. Current advances in the development of abrasive tools and investigation of diamond abrasive machining processes (materials science approach). Journal of Superhard Materials, 2018, 40(5): 348-354. https://doi.org/10.3103/S1063457618050064.

[2] S J Zhang, S To, G Q Zhang. Diamond tool wear in ultra-precision machining. International Journal of Advanced Manufacturing Technology, 2017, 88(1-4): 613-641. https://doi.org/10.1007/s00170-016-8751-9.

[3] L Zhou, J C Yin, Y Huang, et al. Essential causes for tool wear of single crystal diamond in ultra-precision cutting of ferrous metals. Diamond and Related Materials, 2018, 86: 29-40. https://doi.org/10.1016/j.diamo nd.2018.04.012

[4] D D Roshon. Electroplated diamond-composite coatings for abrasive wear resistance. IBM Journal of Research and Development, 1978, 22 (6): 681-686. https://doi.org/10.1147/rd.226.0681.

[5] DY Zhang. Diamond grits are orderly arranged through dispensing method thus improving bit performance. Diamond \& Abrasives Engineering, 2017, 37(4): 11-14. https://doi.org/10.13394/j.cnki.jgszz.2017.4.0003. (in Chinese)

[6] W Li, Y H Ren, C F Li, et al. Investigation of machining and wear performance of various diamond micro-grinding tools. International Journal of Advanced Manufacturing Technology, 2019, 106(3-4): 921-935. https://doi. org/10.1007/s00170-019-04610-4.

[7] A K Chattopadhyay, L Chollet, H E Hintermann. On performance of brazed bonded monolayer diamond grinding wheel. CIRP Annals, 1991, 40(1): 347-350. https://doi.org/10.1016/S0007-8506(07)62003-4.

[8] Y Q Yu, X R Tie, G Q Zhang, et al. Comparison of brazed and sintered diamond tools for grinding of stone. Materials Research Innovations, 2014, 18: 869-873. https://doi.org/10.1179/1432891714z.000000000525.

[9] J C Chen, D K Mu, X J Liao, et al. Interfacial microstructure and mechanical properties of synthetic diamond brazed by Ni-Cr-P filler alloy. 
International Journal of Refractory Metals \& Hard Materials, 2018, 74: 52-60. https://doi.org/10.1016/j.jirmhm.2018.03.005.

[10] B J Ma, H D Zhu. A study on induction brazing of diamond grits using both amorphous and crystalline Ni-based filler alloy. International Journal of Advanced Manufacturing Technology, 2016, 86(5-8): 1607-1613. https:// doi.org/10.1016/j.jmatprotec.2006.05.006.

[11] PY Dong, B Huang, J S Zhang, et al. Investigation and improvement of wear nonuniformity of diamond tools in sawing granite. International Journal of Refractory Metals \& Hard Materials, 2019, 83: 104961. https://doi. org/10.1016/j.jirmhm.2019.05.007.

[12] C M Sung. Brazed diamond grid: a revolutionary design for diamond saws. Diamond and Related Materials, 1999, 8(8-9): 1540-1543. https://doi. org/10.1016/s0925-9635(99)00086-2.

[13] CM Sung. Brazed diamond tools by infiltration: US, 6039641. 2001-0204[2020-06-30]. https://www.freepatentsonline.com/6193770.pdf.

[14] G Burkhard, F Rehsteiner, B Schumacher. High efficiency abrasive tool for honing. CIRP Annals, 2002, 51(1): 271-274. https://doi.org/10.1016/s0007 $-8506(07) 61515-7$.

[15] Q P Wu, ZY Ouyang, Y Wang, et al. Precision grinding of engineering ceramic based on the electrolytic dressing of a multi-layer brazed diamond wheel. Diamond and Related Materials, 2019, 100: 107552. https:// doi.org/10.1016/j.diamond.2019.107552.

[16] L M Song, B Xiao, XY Ding, et al. Development of fundamental analysis of the multilayer brazed diamond saw blades. Diamond \& Abrasives Engineering, 2013, 33(3): 1-4. https://doi.org/10.13394/j.cnki.jgszz.2013.03.001. (in (hinese)

[17] F Long, P He, D P Sekulic. Research and development of powder brazing filler metals for diamond tools: A review. Metals, 2018, 8(5): 315. https:// doi.org/10.3390/met8050315.

[18] Q P Wu, Z H Deng, Z Pan, et al. Effect of brazing atmosphere on brazing performance of diamond. Journal of Mechanical Engineering, 2012, 48(4): 51-57. https://doi.org/10.3901/JME.2012.04.051. (in Chinese)

[19] H Park. ARIX-a major advance in diamond segment design. Industrial Diamond Review, 2005, 65: 40-42. https://www.sdcab.se/wp-content/ uploads/2012/08/ARIX-in-Industrial-Diamond-Review-2005.pdf.

[20] L Chen, Y He, Y X Yang, et al. The research status and development trend of additive manufacturing technology. International Journal of Advanced Manufacturing Technology, 2017, 89(9-12): 3651-3660. https://doi. org/10.1007/s00170-016-9335-4.

[21] J Plocher, A Panesar. Review on design and structural optimisation in additive manufacturing: Towards next-generation lightweight structures. Materials and Design, 2019, 183: 108164. https://doi.org/10.1016/.matde s.2019.108164.

[22] Z B Yang, M G Zhang, Z Zhang, et al. A study on diamond grinding wheels with regular grain distribution using additive manufacturing
(AM) technology. Materials and Design, 2016, 104: 292-297. https://doi. org/10.1016/j.matdes.2016.04.104.

[23] Z B Yang, J Hu, K Li, et al. 3D printing of diamond tools for dental ceramics processing. Advanced Engineering Materials, 2017, 20(3): 1700747. https:// doi.org/10.1002/adem.201700747.

[24] R Gåhlin, H Björkman, P Rangsten, et al. Designed abrasive diamond surfaces. Wear, 1999, 233-235: 387-394. https://doi.org/10.1016/s0043 $-1648(99) 00219-7$.

[25] M Bolaños, M Dimiccoli, P Radeva. Toward storytelling from visual lifelogging: An overview. IEEE Transactions on Human-Machine Systems, 2017, 47(1): 77-90. https://doi.org/10.1109/THMS.2016.2616296.

[26] Y P Ma, Q W Li, J Xing, et al. An intelligent object detection and measurement system based on trinocular vision. IEEE Transactions on Circuits and Systems for Video Technology, 2020, 30(3): 711-724. https://doi. org/10.1109/tcsvt.2019.2897482.

[27] B Lin. Research on geometric dimension measurement system of shaft parts based on machine vision. EURASIP Journal on Image and Video Processing, 2018: 101. https://doi.org/10.1186/s13640-018-0339-x.

[28] Y Du, S X Zhou, X J Jing, et al. Damage detection techniques for wind turbine blades: A review. Mechanical Systems and Signal Processing, 2020, 141: 106445. https://doi.org/10.1016/j.ymssp.2019.106445.

[29] J Sun, C Li, X J Wu, et al. An effective method of weld defect detection and classification based on machine vision. IEEE Transactions on Industrial Informatics, 2019, 15(12): 6322-6333. https://doi.org/10.1109/ tii.2019.2896357.

[30] M Schweier, M W Haubold, M F Zaeh. Analysis of spatters in laser welding with beam oscillation: A machine vision approach. CIRP Journal of Manufacturing Science and Technology, 2016, 14: 35-42. https://doi. org/10.1016/j.cirpj.2016.05.004.

[31] S P Diao, X D Chen, J H Luo. Development and experimental evaluation of a $3 d$ vision system for grinding robot. Sensors, 2018, 18(9): 3078. https ://doi.org/10.3390/s18093078.

[32] Y S Zhao, L Gong, Y X Huang, et al. A review of key techniques of visionbased control for harvesting robot. Computers and Electronics in Agriculture, 2016, 127: 311-323. https://doi.org/10.1016/j.compag.2016.06.022.

[33] A Voulodimos, N Doulamis, A Doulamis, et al. Deep learning for computer vision: a brief review. Computational Intelligence and Neuroscience, 2018: 7068349. https://doi.org/10.1155/2018/7068349.

[34] H L Zhang, L Peng, Y K Luo, et al. Rectangular illumination method using LED arrays for machine vision. Applied Optics, 2020, 59(11): 3518-3525. https://doi.org/10.1364/ao.384833.

\section{Submit your manuscript to a SpringerOpen ${ }^{\circ}$ journal and benefit from:}

- Convenient online submission

- Rigorous peer review

- Open access: articles freely available online

- High visibility within the field

- Retaining the copyright to your article

Submit your next manuscript at $\boldsymbol{\nabla}$ springeropen.com 